\title{
QUALIDADE DE VIDA DOS PROFISSIONAIS DE ENFERMAGEM NO AMBIENTE DE TRABALHO
}

\author{
Mariana Nascimento Freire*, Emanuele Rosados Costa** \\ Autor correspondente: Mariana Nascimento Freire - marianafreire0590@gmail.com \\ * Graduada em Enfermagem pela Escola Bahiana de Medicina e Saúde Pública, estagiária da 6a edição Estágio de Vivências \\ do SUS promovido pela Escola Estadual de Saúde Pública da Bahia e mediadora do Estágio de Vivências do SUS promovido \\ pela Escola Estadual de Saúde pública da Bahia, membro da Comissão Estadual de Saúde e Bombeiro Civil pela Academia \\ Águias da Vida. \\ ** Enfermeira. Escola Bahiana de Medicina e Saúde Pública
}

\section{Resumo}

A Qualidade de Vida (QV) corresponde ao nível perceptível dos indivíduos perante a sua posição na vida, incluindo as culturas, sistemas de valores nos quais vivenciam em relação aos objetivos, expectativas, padrões e preocupações. Entretanto, estão presentes no exercício laboral dos profissionais de enfermagem situações que podem ocasionar o desequilíbrio físico, mental e social dos mesmos, contribuindo para o aparecimento de acidentes e doenças relacionadas ao trabalho. O presente estudo tem como objetivo analisar a Qualidade de Vida dos profissionais de enfermagem no ambiente de trabalho. Para tal, utilizou-se uma revisão sistemática da literatura em artigos disponíveis nas bases de dados do SciELO e Lilacs. Constata-se na literatura que existem riscos enfrentados pelos trabaIhadores de enfermagem em seu labor, entre eles destacam-se os riscos químicos, mecânicos, biológicos, ergonômicos e os psíquicos. A análise dessa literatura permitiu concluir que os profissionais mais acometidos a estes processos potencializadores de desgastes são os técnicos de enfermagem, pois prestam os cuidados diretos aos pacientes, porém os enfermeiros também são susceptíveis á alteração da qualidade de vida no trabalho, pois são os que menos se afastam das atividades laborais e além de ser a única categoria que realiza o gerenciamento da assistência de enfermagem.

Palavras-chave: Qualidade de Vida; Cargas de Trabalho; Profissionais de Enfermagem.

\section{NURSES' QUALITY OF LIFE AT THE WORKPLACE}

\begin{abstract}
Quality of life (QV) regards individuals' perceptions towards their position in life, including culture, value systems in which they experience their relation to objectives, expectations, standards and concerns. In the

- Artigo submetido para avaliação em 12/03/2016 e aceito para publicação em 09/06/2016 •
\end{abstract}


workplace, nurses may undergo situations that may cause physical, mental and social imbalance impacting negatively in their quality of life and contributing to the accidents and work-related diseases. This study aims to assess the life quality of nursing professionals in the workplace. For this, we used a systematic review of literature on articles available in SciELO and LILACS databases. It is noted in the literature that there are risks faced by nursing staff in their work, among them stand out the chemical, mechanical, biological, ergonomic and psychological ones. The analysis of the literature indicated that the most likely to be affected by these hazards are nursing technicians, due to the fact that they are in direct contact with the patients that they provide health care for. Nurses are also likely to have their life quality impacted by their workplace routines forthey are the least likely to step away from labor activities in addition to being the only category that performs nursing care.

Kepwords: Quality of Life; Workloads; Nursing Professionals.

\section{INTRODUÇÃO}

Segundo a Organização Mundial da Saúde (OMS), a Qualidade de Vida (QV) corresponde ao nível perceptível dos indivíduos perante a sua posição na vida, incluindo as culturas, sistemas de valores nos quais vivenciam em relação aos objetivos, expectativas, padrões e preocupações. A expressão de QV é utilizada por segmentos da sociedade são aspectos subjetivos e objetivos que se pautam na necessidade do indivíduo em promover a busca do equilíbrio interno e externo.(1)

O conceito de Qualidade de Vida no Trabalho (QVT) foi difundido na atualidade, por meio de revisões de vínculos na estruturação da vida profissional e pessoal dos fatores socioeconômicos originados pelas metas e pressões organizacionais. A QVT pauta-se nas escolhas de bem- estar e percepção do que poderá ser realizado para favorecer a realização de expectativas dos gestores e trabalhadores. ${ }^{(2)}$

O trabalho está centrado no processo de humanização e autonomia dos indivíduos, classificam-se numa categoria intermediária favorecendo o salto ontológico das formas pré-humanas para o ser social. As condições de trabalho estabelecem a promoção da qualidade de vida e são fatores determinantes que ordenam o poder de consumo dos indivíduos. ${ }^{(3)}$
A enfermagem se insere em vários processos como: os objetos, meios, instrumentos e o trabaIho de enfermagem em si. No seu ambiente de labor, o objeto a ser transformado é o corpo humano individual e coletivo inerente aos processos de saúde - doença. Os meios e instrumentos são os equipamentos utilizados nas terapêuticas e o conhecimento é realizado pelos principais agentes: Os Enfermeiros, os Auxiliares e os Técnicos de enfermagem, que no processo de trabalho envolvem-se em uma forma de organização, divisão e relações de trabalho. ${ }^{(4)}$

As relações no ambiente laboral de enfermagem apresentam diferenciações de acordo com o processo de trabalho executado, pode ocasionar processos potencializadores de desgastes, devido aos processos saúde-doença vivenciadas pelos profissionais, que interfere na Qualidade de Vida no Trabalho. ${ }^{(4)}$

As cargas de trabalho são elementos que ao interagirem entre si e o corpo dos profissionais, ocasionam processos de desgastes pelas perdas das potencialidades físicas e psíquicas, determinada pela exposição dos trabalhadores às cargas de trabalho que se classificam em biológicas, físicas, químicas, mecânicas, fisiológicas e psíquicas. As exposições dos profissionais de enfermagem ocorrem duran- 
te a jornada de trabalho, mesmo que não tenham consciência dos riscos propiciados por este ato. ${ }^{(4,5)}$

Os profissionais de enfermagem, inseridos em diversos setores institucionais convivem com diferentes cargas de trabalho, consequentemente o expondo a situações de riscos ocupacionais distintas. No âmbito do trabalho envolvendo a sua complexidade, o trabalhador poderá trilhar o caminho da produção da saúde ou da produção do desgaste. ${ }^{(5)}$

A relação entre fatores de exposição das cargas de trabalho, ocasionam os processos de desgastes físico-psíquicos, potenciais ou manifestados que permitem traçar um perfil característico de agravos aos profissionais de enfermagem, de modo a interferir na qualidade da assistência prestada aos clientes. ${ }^{(4,5)}$

Em relação aos profissionais que podem ser acometidos pelos processos de desgastes físico-psíquicos, os Técnicos e Auxiliares de enfermagem são suscetíveis à alteração de QV, pois prestam os cuidados diretos aos pacientes com diferentes necessidades e complexidades. $\mathrm{O}$ ambiente laboral destes profissionais é insalubre, os turnos são alternados, existe hierarquização, não há a autonomia, evidencia-se a rigidez na carga horária, rotatividade de setores, aumento de esforços físicos, desarticulação de defesa da equipe e exposição a agentes biológicos. ${ }^{(1)}$

O quantitativo de Enfermeiros no ambiente laboral é inferior em relação aos Técnicos e Auxiliares de enfermagem, evidencia-se que os mesmos se afastam menos das atividades laborais, pois é a única categoria que realiza o gerenciamento da assistência de enfermagem e optam na maioria das vezes em trabalhar doentes para não deixar a sua equipe sem uma pessoa de referência. ${ }^{(6)}$

Com evidências em estágios curriculares, foi percebido que os desgastes dos Técnicos e Auxiliares de enfermagem, resultaram em afastamentos por doenças mecânicas e fisiológicas. Estas observações motivaram o estudo sobre como o exercício laboral pode interferir na QV dos profissionais da área. Com os resultados encontrados, será possível fundamentar estratégias de intervenções nas polí- ticas direcionadas à saúde do trabalhador, assegurando uma melhor QV a estes profissionais.

Por conseguinte, o objetivo deste estudo é analisar a Qualidade de Vida dos profissionais de enfermagem no ambiente de trabalho.

\section{METODOLOGIA}

Trata-se de um estudo de revisão sistemática da literatura, acerca da qualidade de vida dos profissionais no ambiente laboral, com o intuito de prevenir agravos à saúde destes trabalhadores, constatando, assim, o conhecimento produzido sobre este tema e obtendo elementos para analisá-lo.

Uma revisão sistemática, assim como outros tipos de estudo de revisão, é uma forma de pesquisa que utiliza como fonte de dados à literatura sobre determinado tema. Esse tipo de investigação disponibiliza um resumo das evidências relacionadas a uma estratégia de intervenção específica, mediante a aplicação de métodos explícitos e sistematizados de busca, apreciação crítica e síntese da informação selecionada. ${ }^{(6)}$

Este artigo procurou responder a pergunta que sustenta esta pesquisa: Se o trabalhador de enfermagem tem como ocupação o cuidado à saúde dos indivíduos, quais os agravos que estes podem sofrer que interferem na Qualidade de Vida dos Trabalhadores e quais categorias de profissionais são mais susceptíveis a estes agravos no ambiente de labor?

A procura por artigos incluiu pesquisas em bases eletrônicas. As bases pesquisadas foram: Literatura Latino-Americana e do Caribe em Ciências da Saúde (Lilacs); Scientific Eletronic Library (SciELO) e Medical Literature Analysis and Retrieval System Online (Medline). Para a busca dos artigos selecionados, foram utilizados as palavras-chaves em português, perante consulta aos Descritores em Ciências da Saúde: qualidade de vida, cargas de trabalho, profissionais de enfermagem. Utilizando como base estes descritores foram encontradas 
45 publicações, sendo considerados 3 publicações duplicadas.

Para selecionar os artigos, inicialmente, realizou-se a leitura das 35 publicações com a meta de apurar a amostra por meio de critérios de inclusão e exclusão. Foram incluídos artigos originais com- pletos, publicados no período compreendido entre os anos de 2004 e 2014, em periódicos nacionais de enfermagem, com qualificação nacional e internacional do sistema de classificação da CAPES. Excluíram-se os artigos inacessíveis e publicações em línguas estrangeiras.

\begin{tabular}{|c|c|c|c|}
\hline BASE DE DADOS & ARTIGOS IDENTIFICADOS & $\begin{array}{c}\text { ARTIGOS PRÉ- } \\
\text { SELECIONADOS }\end{array}$ & $\begin{array}{c}\text { ARTIGOS } \\
\text { SELECIONADOS }\end{array}$ \\
\hline Lilacs & 0 & 0 & 0 \\
\hline SciELO & 45 & 30 & 14 \\
\hline Medline & 0 & 0 & 0 \\
\hline Total & 45 & 30 & 14 \\
\hline
\end{tabular}

Quadro 1 - Distribuição de artigos identificados e selecionados segundo bases de dados Fonte: elaboração própria.

As informações retratadas na pesquisa foram fundamentadas através de autorias já publicadas, ficando então esclarecido de que as ideias foram formuladas para embasar a publicação do estudo em questão, respeitando sempre os direitos dos autores, como assegurado pela lei 9.610/98, capítulo I, art. 22 que garante ao autor os direitos morais e patrimoniais sobre a obra que criou. Entretanto, fica estabelecido que qualquer hipótese de plágio seja desconsiderada.

\section{RESULTADOS E DISCUSSÃO}

\section{CARACTERIZAÇÃO DOS ESTUDOS}

Os estudos quantitativos foram utilizados para o norteamento deste trabalho de revisão sistemáti$\mathrm{ca}$, que permitiu realizar uma análise dos dados referente à qualidade de vida dos profissionais de enfermagem no ambiente de trabalho, aos agravos que mais interferem na qualidade de vida dos profissionais de enfermagem e identificar a categoria de profissionais de enfermagem mais susceptível á alteração de Qualidade de Vida no seu exercício laboral.
De acordo com o ano de publicação, 2002 esteve em constante evidência, com seis artigos publicados. A Revista Latino Americana de Enfermagem (Rev. Lat-am. Enfermagem) foi o periódico com maior número de artigos utilizados, totalizando treze.

\section{A QUALIDADE DE VIDA DOS PROFISSIONAIS DE ENFERMAGEM}

A Qualidade de Vida (QV) é definida como o entendimento do ser humano do seu posicionamento na vida, no sistema de valores e na contextualização da cultura em que é vivenciado concomitantemente aos seus objetivos, expectativas, padrões e preocupações. O conceito de Qualidade de Vida é muito subjetivo, pois cada pessoa tem seus objetivos e experiências de vida. ${ }^{(1)}$

O termo Qualidade de vida remete-se aos resultados de fatores inter-relacionados, que fazem parte do cotidiano do ser humano, somando-se com as esferas pessoais e acontecimentos nas esferas privada e pública, destacando-se a dimensão do trabalho com expressiva significância da vida das pessoas. ${ }^{(2)}$ 
A qualidade de vida no trabalho traz aspectos do ambiente laboral que interfere na saúde e no desempenho do colaborador, além da relação entre a Qualidade de Vida do profissional de enfermagem e o trabalho. É necessário, que haja um entendimento sobre a relação trabalho/saúde. O processo saúde-doença constitui-se por um conjunto de atributos que operam numa sociedade concreta, que produz nos grupos sociais e evidenciam-se os riscos ou potencialidades que podem ser manifestados por doenças ou saúde. ${ }^{(4)}$

Neste contexto, existem duas vertentes para que o indivíduo consiga o seu sustento e de seus semeIhantes. A produção de matéria-prima e tecnologia resulta numa melhor condição de vida e saúde de toda a humanidade, porém esse mesmo processo de melhoria pode ocasionar nos trabalhadores desgastes físicos - psíquicos, perdas e baixa capacidade laboral, proporcionando alterações pessoais e sociais. O trabalho refere a potência e força do ser humano com intuito de alcançar o seu objetivo e ser capaz de ter domínio sobre a natureza. ${ }^{(6)}$

Assim, é possível evidenciar que na QV em enfermagem há o perfil favorável e o destrutivo. No perfil favorável compreende a integração social, a aprendizagem, a formação de uma identidade social e pessoal, o desenvolvimento e o uso de capacidades humanas. O perfil destrutivo compreende-se pela alienação, subordinação, hierarquização, falta de autonomia, exposição á sobrecargas, ocasionando o processo de desgaste, o aumento da rotatividade de profissionais e a desarticulação das coletividades. ${ }^{(6)}$

\section{PRINCIPAIS AGRAVOS QUE}

\section{INTERFEREM NA QUALIDADE DE VIDA DOS PROFISSIONAIS DE ENFERMAGEM}

De acordo com a organização Pan-Americana da Saúde no Brasil, os agravos á saúde dos trabalhadores referidos a segurança, explicita-se por cinco grandes grupos: Físicos- Condições de anormalidades ambientais ou agressões que podem agravar a saúde dos profissionais; Químicos - Substâncias químicas de origem gasosa ou partículas de poeira minerais ou vegetais evidenciados nos processos de trabalho; Biológicos - Microrganismos correlacionados ao ambiente de labor; Ergonômicos e psicossociais - que se observam na organização e gestão do trabalho; Acidentais- ligados a medidas de proteção das máquinas, condições estruturais, ordem e limpeza do ambiente de trabalho, sinalização, rotulagem de produtos e outros que podem ocasionar os acidentes do trabalho. ${ }^{(7)}$

Dentre os resultados apresentados pelos estudos utilizados como embasamento, constatou que as cargas de trabalho a que são expostos os profissionais de saúde, constituem-se em elementos que se interagem, resultando num processo de desgaste, evidenciado pela perda total ou parcial das capacidades corporais e psíquicas, abrangendo os processos biopsíquicos em seu conjunto e, consequentemente, demonstrando as características da sociedade e definindo um perfil patológico do grupo específico. As cargas de trabalho podem ser categorizadas em fisiológicas, físicas, mecânicas, químicas, biológicas e psíquicas. ${ }^{(8)}$

As Cargas Fisiológicas ocasionam processos de desgastes que influenciam negativamente a força de trabalho, por conta das atividades cotidianas dos profissionais de enfermagem, observa-se que as sobrecargas e o uso do corpo como objeto de trabalho expõem o profissional e compromete seu desenvolvimento futuro e a sua qualidade de vida.(9)

As Cargas Físicas são caracterizadas pelos ruídos do ar-condicionado, sons dos instrumentos de trabalhos, aparelhos ligados aos pacientes, estrutura física comprometida; umidade; iluminação inadequada; eletricidade; risco de incêndio e radiação ionizante. Normalmente esta exposição, ocorre pela falta de manutenção dos aparelhos ou mau uso dos mesmos. ${ }^{(7)}$

As Cargas Mecânicas são derivadas principalmente da tecnologia, devido a sua operação ou manutenção, aos materiais disponíveis ou ao próprio objeto de trabalho. Na carga mecânica, quando o trabalhador interage com elas, representam exigências a sua integridade biopsicossocial que 
gera problemáticas como acidentes de trabalho relacionados com contusões, fraturas e hematomas. É bem evidenciado esse processo quando ocorre a ruptura da integridade do profissional: como os ferimentos por perfuro - cortantes que são visíveis pelas cargas biológicas com vírus, e até mesmo letais, como as hepatites B e C, e AIDS. (7)

As Cargas Biológicas são evidenciadas em vários setores desde uma atenção primária até a terciária, nos hospitais é mais comum ver pacientes com doenças infecto contagiosas e a quantidade de profissionais de enfermagem é maior do que é visto, por exemplo, na atenção primária. É esse tipo de carga que todo profissional de saúde está exposto, pois o contato com o paciente que venha a ter doenças infecciosas, infectocontagiosa, secreções, fluidos e materiais contaminados como perfuro cortantes, normalmente é evidenciado e relatado no momento que se presta o cuidado ao paciente. ${ }^{(7)}$

É possível dizer que as cargas psíquicas são responsáveis pelos desgastes físicos e emocionais da equipe de enfermagem além de ser fonte de estresse e está relacionada com todos os elementos do processo de trabalho, ou seja, está ligada com as demais cargas. A principal fonte das cargas psíquicas pode ser observada no processo de organização e divisão do trabalho. ${ }^{(8)}$

Além do aumento do agravo psíquico no ambiente de labor, evidencia-se que os trabalhadores se submetem a demasiadas pressões sociais e psicológicas, concomitantemente com sua vida pessoal. Os fatores que predispõem os acometimentos dos transtornos mentais como a ansiedade e a depressão são as difíceis condições de trabalho e de vida. (9)

Assim, a exposição dos trabalhadores por produtos químicos seja ele sólido, líquido, gasoso, medicamentoso, antisséptico, desinfetantes, entre outros leva a cargas químicas. Este ponto é algo a ser pensado e avaliado, pois muitas vezes os profissionais que estão expostos encontram-se em locais que não propiciam um espaço físico com ventilação adequada, além de poucas informações sobre o manuseio dos materiais e seus possíveis efeitos no or- ganismo. Muitos desses produtos utilizados e inalados pelos profissionais não demonstram efeitos em curto prazo e por isso muitos nem sabem que pode ter sido prejudicado por esse tipo de carga. ${ }^{(7)}$

\section{CATEGORIA DE PROFISSIONAIS DE ENFERMAGEM MAIS SUSCEPTÍVEIS Á ALTERAÇÃO DE QUALIDADE DE VIDA NO SEU EXERCÍCIO LABORAL}

Analisando o ambiente de laboral na categoria de enfermagem, é possível evidenciar que os enfermeiros são em quantidade inferior quando comparados ao número de técnicos e auxiliares de enfermagem. A responsabilidade do enfermeiro é maior, quando relacionada com á dos técnicos e auxiliares de enfermagem, pois além de realizar as atividades administrativas este tem a função de supervisionar o trabalho da enfermagem, esses profissionais se afastam menos das suas funções do que os técnicos e auxiliares de enfermagem, optando em diversas vezes por trabalhar doentes para não deixar sua equipe sem uma pessoa de referência. ${ }^{(6)}$

É possível dizer que os auxiliares e técnicos de enfermagem são os mais acometidos pelas cargas fisiológicas e biológicas, sendo os que mais se desgastam e comprometem sua qualidade de vida no trabalho, por estarem mais próximos dos pacientes prestando o cuidado direto e por conta das suas práticas laborais. Sendo evidenciada essa realidade, é importante promover aos profissionais de enfermagem atividades de promoção e recuperação da saúde, observando as principais cargas que mais os acometem. ${ }^{(10,11)}$

O fator associado ao aumento da ocorrência dos acidentes de trabalho é o ritmo acelerado, que é devido à rapidez dos profissionais de enfermagem realizarem as suas tarefas e passarem a maior parte da sua jornada de trabalho em pé, manipulando equipamentos pesados que necessitem de força. A não utilização dos Equipamentos de Proteção Individual (EPI) e a ausência do tempo de preparo pré-assistencial contribuem para o aparecimento de doenças ocupacionais e acidentes de trabalho. ${ }^{(5,11)}$ 
A promoção de saúde corrobora para que haja ações de capacitação e empoderamento da população, dessa forma o individuo é capaz de exercer o controle da sua saúde, das outras pessoas e do ambiente em que vive, tomando decisões em que possam conduzi-lo para uma saúde adequada. ${ }^{(12)}$

No entanto, é importante que a empresa elabore um programa de promoção de saúde, deve-se realizar por meio do mesmo a identificação do perfil da população de trabalhadores inserida no contexto de trabalho e iniciar o conceito de implantação e controle de programas. ${ }^{(12)}$

Faz-se necessário, que os profissionais conheçam melhor os riscos para a sua saúde, para que não se faça somente a promoção e prevenção á sua saúde, mas também identifiquem os problemas quando os mesmos o apresentem.

Assim, o comprometimento com as ações que promovam condições de trabalho adequadas para toda equipe de enfermagem como políticas de prevenção, tem como objetivo propiciar um ambiente de labor e saúde adequado. Suporte administrativo no qual o gerenciamento possibilite que essas políticas funcionem, relacionamento interpessoal, consciência de divisão do trabalho de forma uniforme que proporcione qualidade de vida e trabalho, minimizando os índices de adoecimento e absenteísmo. ${ }^{(10)}$

\section{CONSIDERAÇÕES FINAIS}

Este presente estudo possibilitou observar que o ambiente laboral propicia diversos riscos á saúde do profissional de enfermagem que interferem na qualidade de vida no trabalho, que podem ser evitados ou reduzidos por meio de medidas de proteção variadas.

Os trabalhadores estão sujeitos aos acidentes de trabalho porque o ambiente profissional oferece riscos biológicos, físicos, químicos, entre outros.

A prática laboral realizada em condições inadequadas, acompanhada da desvalorização do trabaIhador, relações hierárquicas conflituosas em seu ambiente de trabalho, favorece o aparecimento de estresse neste individuo e estes fatores somados ás cargas enfrentadas pelos profissionais de enfermagem resultando-se em elevados índices de absenteísmo, desgastes físicos e emocionais.

Assim, pode-se dizer que profissionais mais acometidos a estes processos potencializadores de desgastes são os técnicos de enfermagem, pois realizam os cuidados diretos aos pacientes. Porém, os enfermeiros também são susceptíveis á alteração da qualidade de vida, pois são os que menos se afastam das atividades laborais e, além, de ser a única categoria que realiza o gerenciamento da assistência de enfermagem.

Dessa forma faz-se importante repensar a estruturas organizacionais dos serviços de saúde sob a perspectiva do trabalhador que, efetivamente é delimitado cotidianamente com as cargas de trabaIho, devendo ser o ponto focal de propostas intervencionistas para as instituições de saúde.

A elaboração de um programa de saúde nas empresas é importante para identificar o perfil da população de trabalhadores, e com isso facilita a implantação e controle de programas. Os profissionais precisam conhecer os riscos para a sua saúde, saber identificar estes riscos e não somente ter o foco na promoção e prevenção à saúde.

As políticas de prevenção proporcionam para a equipe de enfermagem melhores condições de trabalho, promovendo ambiente e uma saúde adequada. Um gerenciamento de qualidade possibilita que as políticas funcionem, além do relacionamento interpessoal e a divisão do trabalho de forma uniforme, minimizando assim, os índices de absenteísmo e adoecimento.

\section{REFERÊNCIAS}

1. Rios KA, Barbosa DA, Belasco AGS. Avaliação de qualidade de vida e depressão de técnicos e auxiliares de enfermagem. Rev Lat Am Enfermagem. 2010;18(3):[O9 telas]. 
2. Schmidt DRC, Paladine M, Biato C, Pais JD, Oliveira AR. Qualidade de vida no trabalho e burnout em trabalhadores de enfermagem de Unidade de Terapia Intensiva. Rev Bras Enferm 2013;66(1):13-7.

3. Fracolli LA, Granja GF.A utilização da categoria processo de trabalho pela enfermagem brasileira:uma análise bibliográfica. Rev Esc Enferm Usp. 2005;39(Esp.):597-602.

4. Rocha SSL, Felli VEA. Qualidade de vida no trabalho docente em enfermagem. Rev Lat Am Enfermagem. 2004;12(1):28-35.

5. Mininel VA, Felli VEA, Silva EJ, Torri Z, Abreu AP, Branco MTA. Cargas de trabalho, processos de desgaste e absenteísmo-doença em enfermagem. Rev Lat Am Enfermagem. 2013;18(3):[O9 telas] .

6. Sampaio RF, Mancini MC. Estudo de revisão sistemática: um guia para síntese criteriosa da evidência científica. Rev Bras Fisioter. 2007;11(1):83-89.

7. Silva LA, Secco IAO, Dalri RCMB, Araújo SA, Romano CC, Silveira SE. Enfermagem do trabalho e ergonomia: prevenção de agravos à saúde. Rev Enferm UERJ. 2011;19(2):317-323.

8. Felli VEA, Tronchin DMR. A qualidade de vida no trabalho e a saúde do trabalhador de enfermagem. In: Kurcgant P,Tronchin DMR, Fugulin FMT, organizadores. Gerenciamento em
Enfermagem. $2^{a}$ ed. Rio de Janeiro: Guanabara Koogan; 2010.

9. Sampaio RF, Mancini MC. Estudo de revisão sistemática: um guia para síntese criteriosa da evidência científica. Rev Bras Fisioter. 2007;11(1):83-89.

1O. Santos El, Valois BRG. Riscos ocupacionais relacionados ao trabalho de enfermagem: revisão integrativa de literatura. Revista Augustus. 2011;32(16):78-89.

11. Sápia T, Felli VEA, Ciampone MHT. Problemas de saúde de trabalhadores de enfermagem em ambulatórios pela exposição à cargas fisiológicas. Acta Paul Enferm. 2009; 6(22):808-8.

12. Casaburi PR. A enfermagem promovendo a qualidade de vida no trabalho In: Luongo J, Freitas GF. Enfermagem do trabalho. São Paulo: Rideel, $2 \mathrm{Ol} 2$.

13. Martinato MCNB, Severo DF, Marchand EAA, Siqueira $\mathrm{HCH}$. Absenteísmo na enfermagem: uma revisão integrativa. Rev Gaúch Enferm. 2010 mar;31(1):160-6.

14. Santana LL, Miranda FMD, Karino ME, Baptista PCP, Felli VEA, Sarquis LMM. Cargas e desgastes de trabalho vivenciados entre trabalhadores de saúde em um hospital de ensino. Rev Gaúch Enferm. 2013;34(1):64-70. 